\title{
Similar results of robotic and uniportal videothoracoscopic surgery for lung cancer treatment
}

\author{
Giulia Veronesi ${ }^{1}$, Stefanos Bonovas ${ }^{2}$ \\ ${ }^{1}$ Division of Thoracic and General Surgery, Humanitas Cancer Center, Milan, Italy; ${ }^{2}$ Department of Biomedical Sciences, Humanitas University, \\ Milan, Italy \\ Correspondence to: Dr. Giulia Veronesi. Head of Robotic Thoracic Surgery Unit, Division of Thoracic and General Surgery, Humanitas Cancer \\ Center, Via Manzoni 56, 20089 Rozzano, Milan, Italy. Email: giulia.veronesi@cancercenter.humanitas.it. \\ Provenance: This is an invited Editorial commissioned by Section Editor Dr. Min Zhang (Department of Thoracic Oncology, The First Affiliated \\ Hospital of Chongqing Medical University, Chongqing, China). \\ Comment on: Yang S, Guo W, Chen X, et al. Early outcomes of robotic versus uniportal video-assisted thoracic surgery for lung cancer: a propensity \\ score-matched study. Eur J Cardiothorac Surg 2017. [Epub ahead of print].
}

Submitted Feb 07, 2018. Accepted for publication Mar 05, 2018.

doi: $10.21037 /$ jtd.2018.03.88

View this article at: http://dx.doi.org/10.21037/jtd.2018.03.88

In a recent publication, Yang et al. report the results of a retrospective analysis of intraoperative and early postoperative outcome data from patients with non-smallcell lung cancer (NSCLC) admitted in their institution from January 2015 to September 2016. Their aim was to compare the robotic-assisted thoracic surgery (RATS) and uniportal video-assisted thoracic surgery (UVATS) techniques for NSCLC treatment. Data from 153 subjects were collected; 76 had undergone RATS, and 77 UVATS. Patients themselves had selected the surgical method, while all surgeries had been carried out by a single surgeon (1).

A propensity score-matched analysis using four factors (i.e., age, gender, tumour size, and operative procedure) was performed. The comparison of the two groups (of 69 NSCLC cases each) did not show any difference in operative time, chest tube duration, postoperative hospital stay, analgesic usage, overall complication rate, or the number of resected lymph nodes. It was shown, however, that RATS is associated with significantly less intraoperative blood loss, and significantly more dissected lymph node stations, as compared to UVATS.

A main limitation of this study stems from its observational nature: it lacks the experimental random allocation of the intervention, which is necessary to optimally test exposure-outcome hypotheses. Importantly, the authors employed a propensity score method (2) to account for potential confounding factors that might influence both group assignment and study outcomes. It is well known, however, that the propensity score matching technique cannot remove hidden biases caused by unmeasured factors (3). Therefore, unadjusted confounding may still exist if unmeasured factors have influenced treatment selection, making it difficult to delineate the true effect of one surgery technique versus the other.

Some other limitations also exist: (I) the authors did not conduct any power and sample size calculation to increase precision, thereby ensuring that the final conclusions about treatment effects are valid. The actual sample size is small, possibly resulting in a type II statistical error. Here, it is important to note that the rate of conversion to thoracotomy was double in UVATS $(5.2 \%)$ versus RATS (2.6\%) (1); however, the numbers were small and their difference not statistically significant; (II) the single center design may compromise the study's external validity (i.e., the generalizability of results to other populations or settings). Moreover, this research is based on the experience of a single surgeon who had performed more than 300 conventional video-assisted thoracic surgery (VATS) lobectomies before the RATS and UVATS, thus an imbalance in technical skills may be possible due to inclusion in the RATS series of initial cases of the learning curve; and (III) several patient-important outcomes, such as the postoperative pain, tumour recurrence, and longterm survival, were not examined. The results of this study 
should, therefore, be interpreted with caution.

The above limitations are discussed not to disparage the authors in any way. On the contrary, their efforts are much appreciated. It is noteworthy that in both RATS and UVATS series reported in the Yang et al.'s article, the complication rates were very low with zero 30-day mortality. These are exceptional results as compared to an early post-treatment mortality rate of approximately $2 \%$ reported in historical series of conventional open lobectomies, especially in a period of intense debate about the use of stereotactic ablative radiotherapy (SABR) or surgery for patients with early stage NSCLC $(4,5)$. In fact, such comparisons should be repeated considering as surgical cases those treated with minimally invasive approaches.

Regarding the technical aspects of RATS, the authors adopted a 5-port technique with carbon dioxide insufflation, which is a widely used robotic technique, first described by Cerfolio et al. in 2011 (6). Another widespread robotic technique is that described by Park et al. and modified by our group $(7,8)$, which is characterized by the use of an anterior utility incision since the beginning of the procedure, four accesses instead of five, and no routine use of carbon dioxide insufflation.

On the other hand, UVATS has become very popular during the last 10 years and many expert VATS surgeons have adopted it, mainly because it offers an alignment between the vision of the surgical field and the tools insertion, avoiding the mirror effects and limitations related to the different angle between the surgeon view and tip of the instruments (9). UVATS has been described as advantageous for surgeons; however, the reduced working space, the uncomfortable position of the surgeon and the conflicts between camera and tools have convinced many UVATS surgeons to try the robotic system, and some of them to adopt this new approach. Several technical advantages of the robotic procedures have been underlined, such as the stable camera platform, the improved view, and the precision of movements. On the other hand, the absence of tactile feedback and the lack of a variety of tools like proper vascular clamps, remain the main limitations of robotic technology.

While we agree with the conclusion of the article by Yang et al. (1) that the two minimally invasive surgical techniques are feasible and safe for NSCLC treatment, we expect that more advantages of the robotic approach will be underlined when robotic and video-assisted approaches will be compared in treatment of more complex cases, such as difficult segments or advanced disease after chemotherapy or radiotherapy (10). The extension of indication of manual minimally invasive surgery (M-MIS) in case of locally advanced N2 disease is not obvious as stated in the consensus paper on indication of VATS (11).

Postoperative pain and quality of life remain two of the most important outcomes to be evaluated, but their valid assessment requires prospective study designs, and the use of appropriate questionnaires taking into consideration different methods of pain treatment applied during and after surgery. One relevant case-control study (12) compared robotic versus VATS with regards to postoperative pain and quality of life, showing statistically significant benefits of the robotic approach for postoperative pain reduction and earlier return to usual activities, though the number of analyzed cases was rather small.

Our comments aim to highlight that further highquality research on the topic is warranted. Ideally, one large prospective multicenter randomised trial comparing RATS to VATS should be initiated, including the UVATS technique. Few centers of the ROMAN studies, a multicenter randomised trial comparing manual versus robotic video-assisted lobectomy, include surgeons that routinely adopt UVATS for major resection, thus some data will be available after the closure of this study. Also welldesigned, adequately-powered, real-world studies reporting patient-important outcomes should be very welcome to increase the quantity and the quality of clinical evidence on minimally invasive thoracic surgery techniques.

\section{Acknowledgements}

None.

\section{Footnote}

Conflicts of Interest: G Veronesi had financial relationship with Medtronic, Abmedica and Versurgical for consultation and proctoring. The other author has no conflicts of interest to declare.

\section{References}

1. Yang S, Guo W, Chen X, et al. Early outcomes of robotic versus uniportal video-assisted thoracic surgery for lung cancer: a propensity score-matched study. Eur J Cardiothorac Surg 2017. [Epub ahead of print].

2. Rosenbaum PR, Rubin DB. The central role of the propensity score in observational studies for causal effects. 
Biometrika 1983;70:41-55.

3. Stukel TA, Fisher ES, Wennberg DE, et al. Analysis of observational studies in the presence of treatment selection bias: effects of invasive cardiac management on AMI survival using propensity score and instrumental variable methods. JAMA 2007;297:278-85.

4. Stokes WA, Bronsert MR, Meguid RA, et al. Posttreatment mortality after surgery and stereotactic body radiotherapy for early-stage non-small-cell lung cancer. J Clin Oncol 2018;36:642-51.

5. Chang JY, Senan S, Paul MA, et al. Stereotactic ablative radiotherapy versus lobectomy for operable stage I nonsmall-cell lung cancer: a pooled analysis of two randomised trials. Lancet Oncol 2015;16:630-7.

6. Cerfolio RJ, Bryant AS, Skylizard L, et al. Initial consecutive experience of completely portal robotic pulmonary resection with 4 arms. J Thorac Cardiovasc Surg 2011;142:740-6.

7. Park BJ, Flores RM, Rusch VW. Robotic assistance for video-assisted thoracic surgical lobectomy: technique and initial results. J Thorac Cardiovasc Surg 2006;131:54-9.

Cite this article as: Veronesi G, Bonovas S. Similar results of robotic and uniportal videothoracoscopic surgery for lung cancer treatment. J Thorac Dis 2018;10(Suppl 9):S1064-S1066. doi: $10.21037 /$ jtd.2018.03.88
8. Veronesi G, Galetta D, Maisonneuve P, et al. Four-arm robotic lobectomy for the treatment of early-stage lung cancer. J Thorac Cardiovasc Surg 2010;140:19-25.

9. Gonzalez-Rivas D, Paradela M, Fernandez R, et al. Uniportal video-assisted thoracoscopic lobectomy: two years of experience. Ann Thorac Surg 2013;95:426-32.

10. Park BJ, Yang HX, Woo KM, et al. Minimally invasive (robotic assisted thoracic surgery and video-assisted thoracic surgery) lobectomy for the treatment of locally advanced non-small cell lung cancer. J Thorac Dis 2016;8:S406-13.

11. Yan TD, Cao C, D'Amico TA, et al. International VATS Lobectomy Consensus Group. Video-assisted thoracoscopic surgery lobectomy at 20 years: a consensus statement. Eur J Cardiothorac Surg 2014;45:633-9.

12. Louie BE, Farivar AS, Aye RW, et al. Early experience with robotic lung resection results in similar operative outcomes and morbidity when compared with matched video assisted thoracoscopic surgery cases. Ann Thorac Surg 2012;93:1598-604; discussion 1604-5. 